\title{
Algebraic properties for some permutation statistics
}

\author{
Vincent Vong ${ }^{1}$ \\ ${ }^{1}$ Laboratoire d'Informatique Gaspard Monge, Université Paris-Est Marne-la-Vallée, 5 Boulevard Descartes, Champs- \\ sur-Marne, 77454 Marne-la-Vallée cedex 2, France
}

\begin{abstract}
In this article, we study some quotient sets on permutations built from peaks, valleys, double rises and double descents. One part is dedicated to the enumeration of the cosets using the bijection of Françon-Viennot which is a bijection between permutations and the so-called Laguerre histories. Then we study the algebraic properties of these quotient sets. After having shown that some of them give rise to quotient algebras of FQSym, we prove that they are also free.

Résumé. Dans cet article, on étudie certains quotients de l'ensemble des permutations construits à partir des pics, vallées, double montées, double descentes. Une des parties est consacrée au calcul des cardinaux des ensembles quotients. Pour cela, on utilisera la bijection de Françon-Viennot, qui transforme les permutations en histoires de Laguerre. Puis, nous nous intéressons aux propriétés algébriques de ces quotients. En particulier, après avoir montré que certains d'entre eux sont des algèbres quotients de FQSym, on montre qu'ils sont libres.
\end{abstract}

Keywords: Laguerre histories, Free quasi-symmetric functions, quotient algebra, increasing binary trees

\section{Introduction}

One of the goals of algebraic combinatorics is to study operations on discrete structures, and relations between these structures. The set of permutations has provided many recent results in combinatorics and in algebra. In particular, an algebra based on permutations, called FQSym ([11], [1]), is a noncommutative generalization of the algebra of symmetric functions. It contains the theory of the noncommutative symmetric functions ([4]) and many other algebras. Some of them are described thanks to equivalence relations on permutations, as NCSF (having the same descents [7]), PBT (having the same binary search tree [6]), or FSym (having the same $P$-symbol in the $R S K$ algorithm [12]). The formalism of FQSym has enabled to find simple proofs of some results, as e.g., the Littlewood-Richardson rule ([9]), the construction of the peak algebra $([7])$, the representation theory of the 0-Hecke algebra $([8])$.

On the enumerative part, in 1979, Françon and Viennot established a bijection between permutations and Laguerre histories ([3]). These objects encode in a natural way some statistics on permutations: peaks, valleys, double rises, and double descents. This bijection has been used in many occasions, and was one of the building blocks of the combinatorial theory of orthogonal polynomials along with [2]. Since this bijection concerns permutations, hence elements indexing the basis of FQSym, one natural question is how Laguerre histories can be understood in this algebraic framework.

subm. to DMTCS (C) by the authors Discrete Mathematics and Theoretical Computer Science (DMTCS), Nancy, France 
A first approach is given here: indeed, some statistics have enabled us to build some subalgebras and/or quotient algebras of FQSym. So one can ask whether among the four statistics peaks, valleys, double rises, and double descents, some statistics allow one to build quotient algebras. In our case, we will see that some of them we can indeed construct some quotient algebras of FQSym.

We begin by recalling some classical definitions and the bijection of Françon-Viennot. Then we compute the cardinality of the different quotient sets. Finally, the algebraic aspect is tackled: we first study sets defining quotient algebras of FQSym, and then show that these algebras are free.

\section{Definitions and bijection of Françon-Viennot}

\subsection{Some statistics on permutations}

Let us denote by $\mathfrak{S}_{n}$ the set of permutations of size $n$ and by $\mathfrak{S}=\cup_{n} \mathfrak{S}_{n}$. Let $\sigma$ be in $\mathfrak{S}_{n}$, and $i$ in $[1, n]$. Since we want to define the type of any value by comparing it to its left and right neighbours, we need to fix some conventions for $\sigma(0)$ and $\sigma(n+1)$. Up to simple transforms, there are only two distinct conventions: $\sigma(0)=0, \sigma(n+1)=0$, and $\sigma(0)=0, \sigma(n+1)=\infty$. Then $\sigma(i)$ is called:

$$
\begin{array}{ll}
\text {-a peak } & \text { if } \sigma(i-1)<\sigma(i)>\sigma(i+1), \\
\text {-a valley } & \text { if } \sigma(i-1)>\sigma(i)<\sigma(i+1), \\
\text {-a double rise } & \text { if } \sigma(i-1)<\sigma(i)<\sigma(i+1), \\
\text {-a double descent } & \text { if } \sigma(i-1)>\sigma(i)>\sigma(i+1) .
\end{array}
$$

For example, with the convention $0-0$, the permutation 27416358 has the peaks set $\{7,6,8\}$, the valleys set $\{1,3\}$, the double rises set $\{2,5\}$, and the double descents set $\{4\}$ (graphical representation of definitions on Figure 11. Note that these statistics extend directly to words over $\mathbb{N}$ with no repeating letters.

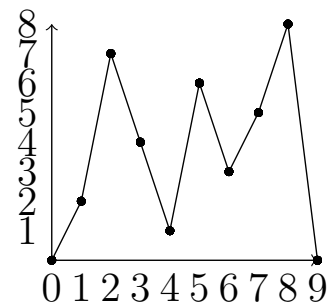

Figure 1: Graphical representation of the permutation 27416358.

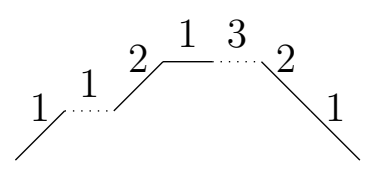

Figure 2: A Laguerre history of size 7.

From now on, we shall work with the convention $0-0$, the case $0-\infty$ being essentially the same. Indeed, by embedding $\mathfrak{S}_{n}$ in $\mathfrak{S}_{n+1}$ by adding $n+1$ as fixed point for a permutation of size $n$, we come back to the case $0-0$. By slightly adapting the proofs, all results of the case $0-0$ transpose to the case $0-\infty$. 


\subsection{The algebra of free quasi-symmetric functions FQSym}

Let $w$ be a word over $\mathbb{N}$. Recall that the shifted word $w[k]$ is the word in which each letter of $w$ is shifted by $k$ : if $w=w_{1} \cdots w_{n}$, then $w[k]=\left(w_{1}+k\right) \cdots\left(w_{n}+k\right)$. The shuffle product is defined by induction as follows:

- $\epsilon \amalg a=a \amalg \epsilon=a$,

- $w=a u, v=b t, w \amalg v=a(u \amalg v)+b(w \amalg t)$, where $a$ and $b$ are letters.

The shifted shuffle of $\sigma \in \mathfrak{S}_{n}$ and $\tau$ is denoted by $\sigma \varpi \tau$, and is equal to $\sigma \varpi \tau[n]$. For example, the shifted shuffle product of $\sigma=12$ and $\tau=\mathbf{2 1}$ is

$$
\sigma \bar{\varpi}=12 \mathbf{4 3}+\mathbf{1 4 2 3}+\mathbf{1 4 3 2}+\mathbf{4 1 2 3}+\mathbf{4 1 3 2}+\mathbf{4 3 1 2}
$$

The algebra FQSym is generated by the basis $\left\langle\mathbf{F}_{\sigma}\right\rangle_{\sigma \in \mathfrak{S}}$ and has the following product formula:

$$
\mathbf{F}_{\sigma} \mathbf{F}_{\tau}=\sum_{\nu \epsilon \sigma \bar{\varpi} \tau} \mathbf{F}_{\nu}
$$

More details about FQSym can be found in [1].

\subsection{The bijection of Françon-Viennot}

Let us now introduce the bijection of Françon-Viennot. It is a bijection between permutations and the so-called Laguerre histories. All details can be found in [3].

\subsubsection{Laguerre histories}

A Laguerre history of size $n$ is a positive valued path, beginning at $(0,0)$, ending at $(n, 0)$ staying above the horizontal axis, with four types of steps: $(1,1),(1,-1),(1,0)$, and an another type of horizontal step, $\overline{(1,0)}$. The $i$-th step has an integer value between 1 and $\gamma(i)$, where $\gamma$ is the following function:

$-\gamma(1)=1$

- $\gamma(i+1)=\gamma(i)$ if the $\mathrm{i}$-th step is $\overline{(1,0)}$ or $(1,0)$,

- $\gamma(i+1)=\gamma(i)+1$ if the $\mathrm{i}$-th step is $(1,1)$,

- $\gamma(i+1)=\gamma(i)-1$ if the $\mathrm{i}$-th step is $(1,-1)$.

Figure 2 shows an example of a Laguerre history $h$ of size 7 , where $\overline{(1,0)}$ is represented as a dotted line. On this example, the corresponding function $\gamma$ takes the successive values $1,2,2,3,3,3$, 2. Let $L H(n)$ be the set of Laguerre histories of size $n$.

\subsubsection{The bijection of Françon-Viennot}

Let $h$ be a Laguerre history of size $n-1$ and let us build the corresponding permutation $\sigma$ of size $n$. The algorithm starts with the word $w=\infty$, and we transform this word by applying some rules. One reads the Laguerre history from left to right and, for each step transforms $w$ as follows: if $t$ is the type of the $i$-th step of $h$, and $j$ its weight, the $j$-th $\infty$ of $w$ is replaced by:

$$
\begin{cases}i & \text { if } \mathrm{t} \text { is }(1,-1) \\ \infty i \infty & \text { if } \mathrm{t} \text { is }(1,1) \\ i \infty & \text { if } \mathrm{t} \text { is } \overline{(1,0)} \\ \infty i & \text { if } \mathrm{t} \text { is }(1,0)\end{cases}
$$


Finally, the last $\infty$ is changed into $n$.

For example, the Laguerre history shown Figure 2 gives the following steps:

$$
\begin{gathered}
\infty \stackrel{1}{\rightarrow} \infty 1 \infty \stackrel{2}{\rightarrow} 2 \infty 1 \infty \stackrel{3}{\rightarrow} 2 \infty 1 \infty 3 \infty \stackrel{4}{\rightarrow} 2 \infty 41 \infty 3 \infty \\
\stackrel{5}{\rightarrow} 2 \infty 41 \infty 35 \infty \stackrel{6}{\rightarrow} 2 \infty 41635 \infty \stackrel{7}{\rightarrow} 2741635 \infty \stackrel{8}{\rightarrow} 27416358 .
\end{gathered}
$$

So its corresponding permutation is $\sigma=27416358$. Since each step of the construction is reversible, it induces a bijection between Laguerre histories of size $n-1$ and $\mathfrak{S}_{n}$. Note that with the convention $0-0$, the type of a step of a Laguerre history and the type of the corresponding value in $\sigma$ in one-to-one correspondence. Indeed, the $i$-th step of $h$ is respectively $(1,-1),(1,1), \overline{(1,0)}$, or $(1,0)$, when $i$ is respectively a peak, a valley, a double rise, or a double descent in $\sigma$.

\section{Enumeration of the quotient sets}

Given the four types of statistics on $\mathfrak{S}_{n}$, we construct some quotient sets on $\mathfrak{S}_{n}$ as follows: consider a partition $\left(A_{1}, \cdots, A_{p}\right)$ of $\{\mathrm{P}, \mathrm{V}, \mathrm{Dr}, \mathrm{Dd}\}$. Then two permutations belong to the same class if and only if they have identical unions of sets of statistics for all the $A_{i}$. For example, if we choose $(\mathrm{P}, \mathrm{V}, \mathrm{Dr}, \mathrm{Dd})$, the equivalence relation consists in having the same peaks, valleys, double rises and double descents. At $n=3$, there are five equivalence classes, 213 and 312 belonging to the same class. If we choose to regroup peaks, valleys and double descents, the equivalence relation consists in having the same double rises set, and we will denote it by $(\mathrm{P} \cup \mathrm{V} \cup \mathrm{Dd}$, Dr). For example, 13245 and 14532 are in the same class for the second relation, but not for the first one. Indeed, the four sets respectively are $(\{3,5\},\{2\},\{1,4\}$, $\varnothing)$ and $(\{5\}, \varnothing,\{1,4\},\{2,3\})$. So the permutations do not belong to the same (P, V, Dr, Dd) class but they do belong to same $(\mathrm{P} \cup \mathrm{V} \cup \mathrm{Dd}$, Dr) since both sets are $(\{2,3,5\},\{1,4\})$.

Thanks to the bijection of Françon-Viennot, each type of statistics is interpreted as a type of step. So two permutations of size $n$ are in the same class for (P, V, Dr, Dd) if and only if they have the same unvalued Laguerre histories of size $n-1$, which are the Motzkin paths with two types of horizontal steps. Since each type of step represents one type of statistics, and since $(\mathrm{P}, \mathrm{V}, \mathrm{Dr}, \mathrm{Dd})$ is represented by the unvalued Laguerre histories, the other quotients are obtained by identifying the corresponding types of steps on the unvalued Laguerre histories. For example, if we identify the peaks with the valleys, we identify the steps $(1,-1)$ and $(1,1)$ in the Laguerre histories.

\subsection{The quotient set $(P, V, D r, D d)$}

Thanks to the bijection, (P, V, Dr, Dd) is in bijection with the unvalued Laguerre histories of size $n-1$, and hence is enumerated by the $n$-th Catalan number. The full proof is presented in [3].

\subsection{The quotient set (P, $V, D r \cup D d)$}

In this case, the two types of horizontal steps are identified. Therefore, (P, V, Dr $\cup \mathrm{Dd})$ has a one-toone correspondence with the Motzkin paths of size $n-1$. The representatives are given by the Laguerre histories with all horizontal steps equal to $(1,0)$. 


\subsection{The quotient set $(P \cup V, D r, D d)$}

In this case, the type of steps $(1,1)$ and $(1,-1)$ are identified. Note that in an unvalued Laguerre history, there is always the same number $k$ of steps $(1,1)$ and $(1,-1)$. Let $k$ be an integer between 0 and $\frac{n-1}{2}$. Since the horizontal steps can be anywhere, we have to select $n-1-2 l$ steps in the set $\{1, \cdots, n-1\}$. These steps may be $\overline{(1,0)}$ or $(1,0)$, hence two choices. So we get the following formula for the number of classes:

$$
\sum_{k=0}^{\frac{n-1}{2}}\left(\begin{array}{c}
n-1 \\
n-1-2 k
\end{array}\right) 2^{n-1-2 k}=\sum_{k=0}^{\frac{n-1}{2}}\left(\begin{array}{c}
n-1 \\
2 k
\end{array}\right) 2^{n-1-2 k}=\frac{(2+1)^{n-1}+(2-1)^{n-1}}{2} .
$$

Thus, the number of classes is $\frac{3^{n-1}+1}{2}$. Let us give one representative of each class in term of unvalued Laguerre history. If there are $n-1-2 l$ horizontal steps, there are $2 k$ steps which are not. We take the first such $k$ steps to be of type $(1,1)$, and the others to be of type $(1,-1)$.

\subsection{The quotient sets $(P \cup V \cup D d$, Dr) and $(P \cup V \cup D r, D d)$}

Reversing dotted and not dotted horizontal steps is an involution on unvalued Laguerre histories. In terms of types of statistics, it sends two permutations having the same double rises into two permutations having the same double descents and vice versa, and preserves the peaks and the valleys. As a consequence, these two quotients are in bijection. Therefore, we only study the case of $(\mathrm{P} \cup \mathrm{V} \cup \mathrm{Dd}$, Dr). Here, for each step, we can decide if it is or not a dotted horizontal step. So we have at most $2^{n-1}$ classes. Conversely, let us choose the dotted horizontal steps. Then the other steps can be taken as non dotted horizontal steps, which gives an unvalued Laguerre history. So there are $2^{n-1}$ classes.

\subsection{The quotient sets $(P, D r \cup V \cup D d)$ and $(V, D r \cup P \cup D d)$}

First, we observe that reading the unvalued Laguerre histories right to left is an involution. In terms of types of statistics, it sends two permutations having the same peaks into two permutations having the same valleys, and vice versa, and preserves the double rises and double descents. So, the quotient sets $(\mathrm{P}, \operatorname{Dr} \cup$ $\mathrm{V} \cup \mathrm{Dd})$ and $(\mathrm{V}, \mathrm{Dr} \cup \mathrm{P} \cup \mathrm{Dd})$ are in bijection. Now, for $(\mathrm{P}, \operatorname{Dr} \cup \mathrm{V} \cup \mathrm{Dd})$, identify the steps $\overline{(1,0)},(1,0)$, and $(1,1)$. We then obtain a left factor of a Dyck path of length $n-1$. Conversely, given a left factor of a Dyck path, if there are $k$ steps equal to $(1,-1)$, we keep the $k$ first steps equal to $(1,1)$, and change the other steps equal to $(1,1)$ into $\overline{(1,0)}$. We then get an unvalued Laguerre history. Thus the equivalence classes are in bijection with the left Dyck factors, which are enumerated by the central binomial of $n-1$ [13] $(A 001405)$.

\subsection{The quotient sets $(P, D r, V \cup D d),(P, D d, V \cup D r),(P \cup D d, V, D r)$ and $(P \cup$ $D r, V, D d)$}

With the involutions consisting in reading from right to left or reversing dotted and not dotted horizontal steps, these four quotient sets are in bijection. So, it is enough to study the case (P, Dr, V $\cup$ Dd). Here, the type of steps $(1,1)$ and $(1,0)$ are identified with the step $(1,1)$. So we obtain a left factor of a Motzkin path of size $n-1$. Conversely, if we take a left factor of a Motzkin path, if there are $k$ steps of type $(1,-1)$, we keep the first $k$ steps of type $(1,1)$, and change the others into $\overline{(1,0)}$. So we obtain an unvalued Laguerre history for each left factor of a Motzkin path. So $(\mathrm{P}, \mathrm{Dr}, \mathrm{V} \cup \mathrm{Dd})$ is enumerated by the left factors of Motzkin paths of size $n-1$ which are in bijection with the directed animals of size $n-1$ [13] $(A 005773)$. 


\subsection{The quotient set $(P \cup V, D r \cup D d)$}

Here, the steps $(1,-1)$ and $(1,1)$ are identified. We also identify the step $\overline{(1,0)}$ with the step $(1,0)$. By the same arguments as in the case $\left(\mathrm{P} \cup \mathrm{V}, \mathrm{Dr}\right.$, Dd), we show that the number of classes is $2^{n-2}$.

\subsection{The quotient sets $(P \cup D r, V \cup D d)$ and $(P \cup D d, V \cup D r)$}

By reading from right to left, these two quotient sets are in bijection. Here, we are identifying $(1,-1)$ with $\overline{(1,0)}$, and $(1,1)$ with $(1,0)$. So the number of classes is smaller than or equal to $2^{n-1}$. Since the representatives are given by the paths without $(1,1)$ and $(1,-1)$, the number of classes is indeed $2^{n-1}$.

\section{The quotient algebras of FQSym}

In recent papers, many quotients of $\mathbf{F Q S y m}$ have been constructed from statistics on permutations ([5], [12], [7]). For example, the algebra QSym is the quotient algebra of $\mathbf{F Q S y m}$ where $\mathbf{F}_{\sigma}$ and $\mathbf{F}_{\tau}$ are identified if $\sigma$ and $\tau$ have the same descents. Since we have built some quotient sets in $\mathfrak{S}_{n}$ for each $n$, it is natural to check if there are some quotient algebras in FQSym induced by these equivalence relations. To this aim, we first recall some notions on increasing binary trees. Indeed, our statistics interpret directly on these combinatorial structures. Then we will study all cases beginning with the quotient set $(\mathrm{P}, \mathrm{V}, \mathrm{Dr}$, $\mathrm{Dd})$.

\subsection{Increasing binary trees}

The notion of increasing binary trees appears in [2] as tournament trees. Let $\mathbf{A}$ be a totally ordered alphabet. Let $w$ be a word on $\mathbf{A}$, with no repeated letters. If $w$ is the empty word (denoted by $\epsilon$ ), then the corresponding tree (denoted by $T(w)$ ) is a leaf. Otherwise, we write $w=w_{1} a w_{2}$, where $a$ is the smallest letter in $w$. The corresponding increasing binary tree is recursively built as follows: the letter $a$ is the root, the left subtree is the increasing binary tree associated with $w_{1}$, and the right subtree is the increasing binary tree associated with $w_{2}$. Note that here an increasing binary tree is a complete binary tree, where all internal nodes are labelled, and the leaves are not.

\subsubsection{The grafting operation}

Let $T$ and $T^{\prime}$ be two trees, let $l$ be a leaf of $T$. The graft of $T^{\prime}$ on $T$ at position $l$ is the substitution of $l$ by $T^{\prime}$.

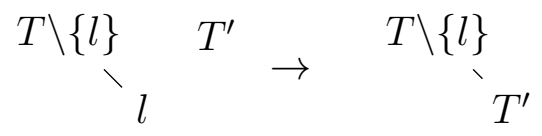

\subsubsection{Increasing binary trees and shifted shuffle}

Let $\sigma$ and $\tau$ be two permutations, and $w$ in $\sigma \bar{\Psi} \tau$. We can write $w$ as: $w=\tau^{(1)} \sigma_{1} \cdots \sigma_{n} \tau^{(n+1)}$, where $\tau[n]=\tau^{(1)} \cdots \tau^{(n+1)}$, and $\tau^{(i)}$ may be an empty word. Then $T(w)$ is the tree built as follows: in the tree $T(\sigma)$, graft the tree of the word $\tau^{(i)}$ at its $i$-th leaf (in the infix order) .

Conversely, if we decompose $\tau[n]=\tau^{(1)} \cdots \tau^{(n+1)}$, with $\tau^{(i)}$ a factor of $\tau[n]$ (maybe empty), and graft at the $i$-th leaf (in the infix order) of $T(\sigma)$ the tree $T\left(\tau^{(i)}\right)$, we obtain an increasing binary tree $T^{\prime}$. By reading $T^{\prime}$ in infix order, we get a $w$ which is in $\sigma \bar{\varpi} \tau$. or example, let $\bar{\sigma}=\overline{2413}, \tau=3.2 .14$. The 
word $w=27416358$ decomposes as $\overline{2} 7 \overline{41} 6 \overline{3} 58$ in $\sigma \bar{\Psi} \tau$ (the empty words are forgotten) and $T(w)$ is represented igure 3

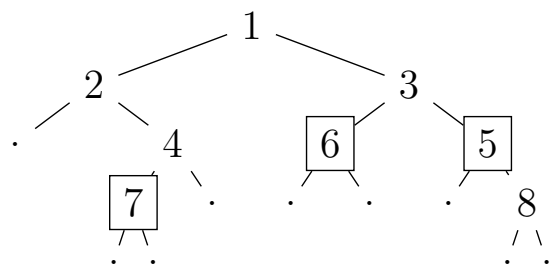

Figure 3: The increasing binary tree of 27416358.

\subsubsection{Increasing binary trees and permutations}

Let us recall that there is a classical bijection between the permutations of size $n$, and the complete increasing binary trees with $n$ internal nodes labelled by $\{1, \cdots, n\}$. A permutation $\sigma$ is sent to its increasing binary tree. Conversely, by reading an increasing binary tree in infix order, we obtain its corresponding permutation. Let us see how the types of statistics are interpreted in the increasing binary tree. Let $\sigma$ be in $\mathfrak{S}_{n}, i$ a letter in $\sigma$, and $T(\sigma)$ the corresponding increasing tree. Note that depending on $i$ being a peak, a valley, a double rise, or a double descent in $\sigma$, the node $i$ in $T(\sigma)$ has respectively zero labelled child, two labelled children, one labelled child to the left or to the right (see the exemple Figure 3 .

\subsection{The quotient algebras of FQSym}

Let us consider one equivalence relation $\sim$ in $\mathfrak{S}$. This relation induces a quotient of FQSym, by identifying $\mathbf{F}_{\sigma}$ and $\mathbf{F}_{\tau}$ if $\sigma \sim \tau$. Proving that this quotient is well-defined is equivalent to prove that if $\sigma$ and $\tau$ are equivalent then for all $s$ in $\mathfrak{S}$, there exists a bijection $\phi$ between $\sigma \bar{\varpi} s$ and $\tau \bar{\Psi} s$, a bijection $\psi$ between $s \bar{\varpi} \sigma$ and $s \bar{\varpi} \tau$ such that each element in $\sigma \bar{\varpi} s$ or in $s \bar{\varpi} \sigma$ are respectively equivalent to their image by $\phi$ or $\psi$.

\subsubsection{The quotient by the four types of statistics}

In this Section, we write $\sigma \sim \tau$ if $\sigma$ and $\tau$ belong to the same (P, V, Dr, Dd) class. In terms of trees, $\sigma \sim \tau$ if and only if for each labelled node of $T(\sigma)$ and $T(\tau)$, the left and right children are of same type (labelled or not). Now, let $w$ be in $\sigma \bar{\Psi} s$.

Construction of $\phi$ : The word $w$ decomposes in the following form: $w=s^{(1)} \sigma_{1} \cdots \sigma_{n} s^{(n+1)}$, with $s[n]=s^{(1)} \cdots s^{(n+1)}$. Denote by $\left(f_{1}, \cdots, f_{k}\right)$ the non-empty factors among the $s^{(i)}$ in order. So we have, $s[n]=f_{1} \cdots f_{k}$.

Since $w$ is in $\sigma \bar{\Psi} s$, we know that $T(w)$ is obtained by grafting at the $i$-th leaf the tree $T\left(s^{(i)}\right)$ (see Section 4.1.2. Let us mark the leaves where the $T\left(f_{j}\right)$ are grafted and denote by $p_{j}$ the father of the leaf where $T\left(f_{j}\right)$ is grafted (two $p_{j}$ may be equal). Note that $T\left(f_{j}\right)$ may graft at the left or right child of $p_{j}$.

Since $\sigma$ and $\tau$ have the same four statistics, all nodes $p_{j}$ in $T(\tau)$ are of the same type as in $T(\sigma)$. So the left and right children of $p_{j}$ are the same type (labelled or not) in $T(\sigma)$ and $T(\tau)$. Let us then mark in $T(\tau)$ the children of $p_{j}$ where there is a graft of a $T\left(f_{j}\right)$ in $T(\sigma)$. Now, we graft at the $i$-th marked 


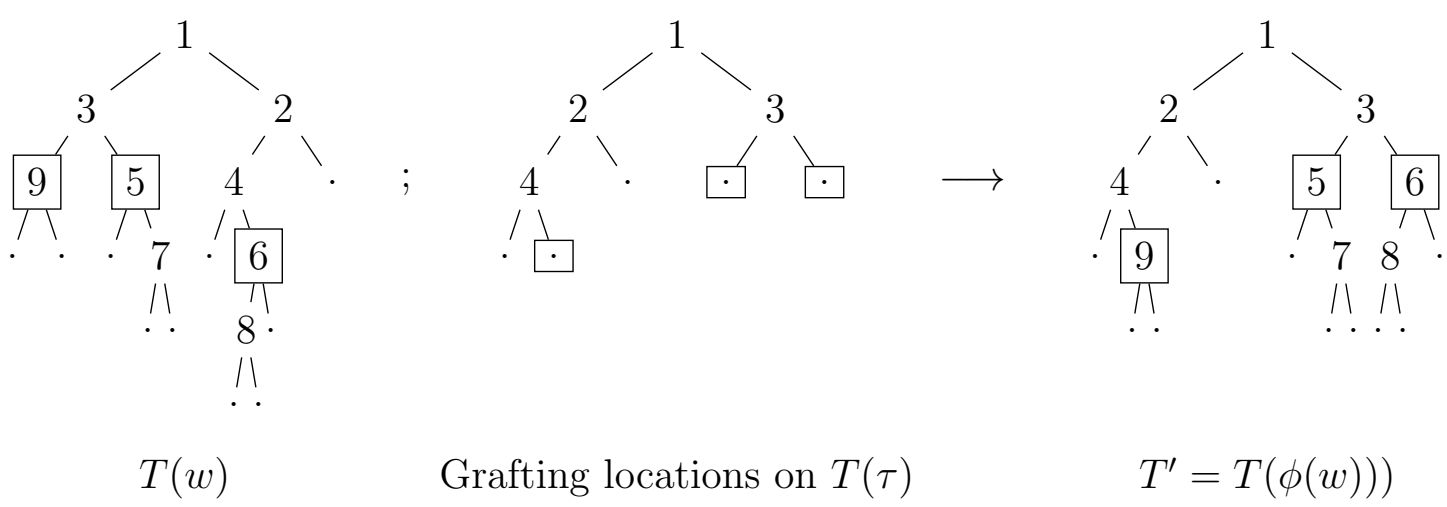

Figure 4: Construction of $T^{\prime}$ with $\sigma=3142, \tau=4213, s=51342$, and $w=935714862$.

leaf of $T(\tau)$ the tree $T\left(f_{i}\right)$. Let $T^{\prime}$ be this tree. By construction, the $p_{j}$ have the same type in $T(w)$ and $T^{\prime}$. Moreover, the other labelled nodes do not change type between type $T(\sigma), T(s)$ and $T^{\prime}$. So each labelled node of $T(w)$ and $T^{\prime}$ have same type. So the permutation $\phi(w)$ associated with $T^{\prime}$ is in the same equivalence class as $w$, and belongs to $\tau \bar{\Psi} s$.

If we apply the same algorithm on $\phi(w)$, exchanging the role of $\tau$ and $\sigma$, we find back $w$. So this operation is a bijection between $\sigma \bar{\Psi} s$ and $\tau \bar{\varpi}$. Here is an example of computation of $\phi$ in Figure 4

Construction of $\psi$ : The key ingredient in building $\psi$ relies on

Proposition 4.1 let $\sigma \sim \tau$ be two words and write $\sigma$ as a concatenation of $k$ words: $\sigma=f_{1} \cdots f_{k}$. Then there exists a decomposition of $\tau=g_{1} \cdots g_{k}$ such that for each letter a in $f_{i}$, the word $g_{j}$ containing a satisfies that a has same type in $f_{i}$ and $g_{j}$.

\section{Proof:}

Let $a_{i}$ be the smallest letter between the last letter of $f_{i}$ and the first letter of $f_{i+1}$. For each $a_{i}$, we cut $\tau$ at the right of $a_{i}$ if $a_{i}$ is in $f_{i}$ and at the left of $a_{i}$ otherwise. Thus, we obtain a decomposition of $\tau$ of the form $g_{1} \cdots g_{k}$. Note that the letters that change type between $\tau$ and the $g_{j}$ are exactly the $a_{i}$. So all but the $a_{i}$ have same type in the $f_{i}$ and $g_{j}$ : their type is preserved from $f_{i}$ to $\sigma$, from $\sigma$ to $\tau$, and from $\tau$ to $g_{j}$. Now, concerning the $a_{i}$, they have same type in $\sigma$ and $\tau$ and it changes in the same way from $\sigma$ to $f_{i}$ than from $\tau$ to $g_{j}$.

For example, let $\sigma=25.7 .1 .34 .6$, and $\tau=3612457$. Here are the different steps in order to find the decomposition of $\tau$ :

$$
3612457 \stackrel{5<7}{\longrightarrow} 361245.7 \stackrel{7>1}{\longrightarrow} 36.1245 .7 \stackrel{1<3}{\longrightarrow} 36.1 .245 .7 \stackrel{4<6}{\longrightarrow} 36.1 .24 .5 .7 .
$$

So the decomposition associated with $\tau$ is 36.1 .24 .5 .7 . Note that the previous statement extends directly to words with no repeated letters of the same evaluation and same type. 
Let us now build the bijection $\psi$. For $w$ in $s \bar{\Psi} \sigma$, we mark the $k$ leaves in $T(s)$ where there is a graft in order to obtain $T(w)$. It corresponds to write $w=\sigma^{(1)} s_{1} \cdots s_{m} \sigma^{(m+1)}$, with $\sigma[m]=\sigma^{(1)} \cdots \sigma^{(m+1)}$ and consider the sequence $\left(f_{1}, \cdots, f_{k}\right)$ of the non-empty factors among the $\sigma^{(i)}$ in order. We apply Proposition 4.1 to $\sigma[m]=f_{1} \cdots f_{k}$ and $\tau[m]$ and obtain a decomposition of $\tau[m]=g_{1} \cdots g_{k}$. Define $T^{\prime}$ as the tree obtained by grafting $T\left(g_{i}\right)$ at the $i$-th marked leaf of $T(s)$. By construction, the labelled nodes in $T(s)$ are of same type in $T(w)$ and $T^{\prime}$. Thanks to Proposition 4.1, the other labelled nodes have also same type. So, by reading $T^{\prime}$ in infix order we obtain a word $\psi(w)$ in $s \bar{\Psi} \tau$, which has same type as $w$.

Note that if we apply this algorithm on $\psi(w)$, exchanging the role of $\sigma$ and $\tau$, we find back $w$. So $\psi$ is a bijection.

Here is an example of computation of $\psi$, with $\sigma=4132, \tau=3214, s=51243$, and $w=591682437$ in $s \bar{\varpi} \sigma$. The word $w$ gives the following factorization for $\sigma: 4.13 .2$. Then we factorize $\tau$ as:

$$
3214 \stackrel{4>1}{\longrightarrow} 321.4 \stackrel{3>2}{\longrightarrow} 3.21 .4 \text {. }
$$

So we have the following grafting locations in $T(s)$ and finally the tree $T^{\prime}$ :

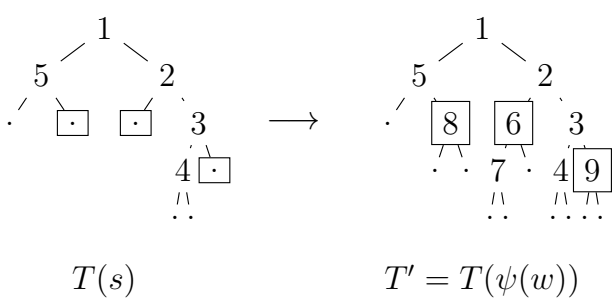

The definitions of both $\phi$ and $\psi$ then proves

Theorem 4.1 The quotient of $\mathbf{F Q S y m}$ by $(P, V, D r, D d)$ is well-defined.

\subsubsection{The other cases}

The case $(\mathrm{P}, \mathrm{V}, \mathrm{Dr} \cup \mathrm{Dd})$ where we consider having the same peaks and valleys is also a well-defined quotient algebra of FQSym. Indeed, in terms of trees, the equivalence concerns nodes having the same number of children which are leaves. So in order to build $\phi$, instead of considering how to graft to the left or to the right, we just graft where it is possible. In order to build $\psi$, we also adapt the factorization: we just cut at the only possible place in order to change the type of a letter.

The other cases studied in the previous part do not give any quotient algebra except $(\mathrm{P} \cup \operatorname{Dr}, \mathrm{V} \cup \mathrm{Dd})$ and $(\mathrm{P} \cup \mathrm{Dd}, \mathrm{V} \cup \mathrm{Dr})$. These cases give back an already known quotient algebra defined in [5].

Let us give some counter-examples for the other cases. In the cases where peaks and valleys are identified, shuffling by 1 on the right does not preserve the statistics. For example, if we take $\sigma=2746351$ and $\tau=2756341$, the words $w=27563481$ and $w^{\prime}=27563841$ are in $\tau \bar{\Psi} 1$, and 4 is a double rise in $w$ and a double descent in $w^{\prime}$, whereas 4 is always a valley in the elements of $\sigma \bar{\Psi} 1$. So the only case where peaks and valleys are identified and where the quotient is well-defined is where all four statistics are identified, that is the case where all elements of $\mathfrak{S}_{n}$ are in the same class, a rather uninteresting case. For $(\mathrm{P} \cup \mathrm{Dr}, \mathrm{V}, \mathrm{Dd})$ and $(\mathrm{V}, \mathrm{P} \cup \operatorname{Dr} \cup \mathrm{Dd})$, consider $\sigma=45312$ and $\tau=53124$. They are in the same class for these two quotients. The permutation $w=645312$ is in $\sigma \bar{W} 1$, and 4 is a valley. But 4 cannot be a valley in the element of $\tau \bar{\varpi} 1$. All other cases are equivalent to the previous ones, up to reversal of the alphabet or mirror image of words. 


\section{The quotient algebras are all free}

After proving that some quotients are well-defined algebras, let us now prove that these quotients are all free. To this aim, we give an isomorphism between a free subalgebra (denoted by B) of FQSym and a quotient (denoted by $\mathbf{C}$ ) having the same sequence of dimensions. We denote by $p$ the projection onto C. The strategy is the following: we consider a basis $B$ of $\mathbf{B}$ and show that the family $(p(b))_{b \in \mathbf{B}}$ spans C. Since in our case we know a basis $C$ of $\mathbf{C}$, we then show that the matrix of $p(B)$ in the basis $C$ is invertible. So $\mathbf{B}$ and $\mathbf{C}$ are isomorphic as algebras.

\subsection{The quotient of FQSym by $(P, V, D r, D d)$}

In this Section, we take $\mathbf{B}=\mathbf{P B T}$ (for more details about PBT, see [10]), $\mathbf{C}=\mathbf{F Q S y m} /(\mathrm{P}, \mathrm{V}, \mathrm{Dr}, \mathrm{Dd}$ ). The projection $p$ is the quotient from FQSym onto $\mathbf{C}$. Let us denote by $\mathfrak{R}=\{\sigma \in \mathfrak{S} \mid \sigma$ avoiding 312 $\}$. Thanks to [3], a basis of $\mathbf{C}$ is given by $\left(p\left(\mathbf{F}_{\sigma}\right)\right)_{\sigma \in \mathfrak{R}}$. We order this family by the inverse lexicographic order, and denote it by $C$.

Recall ([6]) that the $\mathbf{E}$ basis of PBT is given by $\mathbf{E}_{\sigma}=\sum_{\tau>_{P} \sigma} \mathbf{F}_{\tau}$, for $\sigma \in \mathfrak{R}$, where $\geq_{P}$ is the order of the right permutohedron. We denote by $B$ this family ordered by the inverse lexicographic order. Note that PBT and $\mathbf{C}$ have the same sequence of dimensions, which is the Catalan sequence. Moreover, the algebra PBT is free since a family of independent algebraic generators is given by the $\mathbf{E}_{\sigma}$ with $\sigma$ in $\Re$, and ending by 1 . Let $\sigma$ be in $\Re$. We have:

$$
p\left(\mathbf{E}_{\sigma}\right)=\sum_{\tau \geq_{P} \sigma} p\left(\mathbf{F}_{\tau}\right)=\sum_{s \in \mathfrak{R}} c_{s}^{(\sigma)} p\left(\mathbf{F}_{s}\right), \text { where } c_{s}^{(\sigma)}=\left|\left\{\tau \geq_{P} \sigma \mid \tau \sim s\right\}\right| .
$$

Let us admit temporarily the following Proposition.

Proposition 5.1 Let $\sigma$ be in $\mathfrak{R}$. If $\tau$ satisfies $\sigma \leq_{P} \tau$, then the element $\tau^{\prime}$ in $\mathfrak{R}$ which has the same four statistics as $\tau$ satisfies $\sigma \leq_{l e x} \tau^{\prime}$.

Thanks to Proposition 5.1, for $s$ and $\sigma$ in $\Re, c_{s}^{(\sigma)}=0$ if $s<_{l e x} \sigma$. Morever, $c_{\sigma}^{(\sigma)} \geq 1$. So the matrix of $p(B)$ in the basis $C$ is upper triangular with non zero coefficients on the diagonal. So $p(B)$ is a basis of $\mathbf{C}$, and $\mathbf{P B T}$ is isomorphic to $\mathbf{C}$. In particular, $\mathbf{C}$ is free.

Let us prove a slightly more general case than Proposition 5.1. Before stating the result, we first need some notations. Denote by $\mathfrak{X}$ the set of words appearing during the execution of the Françon-Viennot algorithm. It is the set of words that are obtained by inserting some $\infty$ inside permutations, no two $\infty$ consecutive. Let $\mathfrak{X}_{n}$ be the subset of $\mathfrak{X}$ where the permutation is in $\mathfrak{S}_{n}$. Given such an element, working backwards, one easily checks that there is only one way to apply the Françon-Viennot algorithm to get it.

Therefore, given $\tau \in \mathfrak{X}_{n}$, we define two elements: $p(\tau) \in \mathfrak{S}_{n}$ obtained from $\tau$ by erasing the $\infty$ letters and $r(\tau)$ obtained by doing the same replacements in the Françon-Viennot algorithm as to obtain $\tau$ but always on the first $\infty$ sign. Note that in the case where there are no $\infty$ in $\tau$, this algorithm produces the element of the class of $\tau$ avoiding 312 as already proven by Françon and Viennot. We have:

Proposition 5.2 Let $\sigma \in \mathfrak{S}_{n}$ avoiding 312 and $w \in \mathfrak{X}_{n}$ containing at least one $\infty$, such that $\sigma \leq_{P} p(w)$. Then $\sigma \infty \leq_{\text {lex }} r(w)$.

Proof: We make the proof by induction on $n$. For $n=1$, it is obvious. 
Assume that the property holds for all such elements of $\mathfrak{S}_{k}$ with $k \leq n-1$. Let $\sigma \in \mathfrak{S}_{n}$ and in $\mathfrak{R}$. Let $w$ be a word satisfying the statement and let $i$ be the position of 1 in $\sigma$.

Since $\sigma$ avoids $312, \sigma=\sigma_{1} 1 \sigma_{2}$ where $\sigma_{1}$ is a permutation of $\{2, \ldots, i\}$.

If $i=n, \sigma$ ends with 1 , so does $p(w)$, and so does $p\left(w^{\prime}\right)$. Then the property comes by induction on the longest prefixes of $\sigma$ and $w$ not containing 1 .

Otherwise, let us consider the words $\sigma_{(i)}$ and $w_{(i)}$ obtained by stopping the algorithm after only $i$ steps instead of $n$. Then $\sigma_{(i)}=\sigma_{1} 1 \infty$. Since $\sigma \leq_{P} p(w)$, we have $\sigma_{1} 1 \leq_{P} p\left(w_{(i)}\right)$. So the induction hypothesis applies and $\sigma_{(i)} \leq_{\text {lex }} r\left(w_{(i)}\right)$.

If the inequality is strict, then since the $\infty$ in the prefix of size $i$ of $r\left(w_{(i)}\right)$ are only replaced by letters greater than $i$, we deduce that $\sigma_{(i)}<_{\operatorname{lex}} r\left(w_{n}\right)=r(w)$, and then $\sigma \infty<_{\operatorname{lex}} r(w)$. Otherwise, the induction applies on the longest suffixes of $\sigma$ and $w$ not containing 1 and the result follows.

\subsection{The quotient of FQSym by $(P, V, D r \cup D d)$}

In this Section, $\sim$ represents the equivalence of permutations having the same peaks and valleys. Let us denote by $\mathfrak{D}$ the permutations in $\mathfrak{R}$ without double rise. Then $\left(p\left(\mathbf{F}_{\sigma}\right)\right)_{\sigma \in \mathfrak{D}}$ is a basis of $\mathbf{C}=\mathbf{F Q S y m} /(\mathrm{P}, \mathrm{V}, \mathrm{Dr} \cup \mathrm{Dd})$. Let us order this by the inverse lexicographic order, and denote this basis by $C$. In that case, let $\mathbf{B}$ be the subalgebra generated by the family $\left(\mathbf{E}_{\sigma}\right)_{\sigma \in \mathfrak{D}}$. Note that this algebra has the same sequence of dimensions as $\mathbf{C}$. Moreover, $\mathbf{B}$ is free, and a family of independent algebraic generators is given by the $E_{\sigma}$, with $\sigma$ in $\mathfrak{D}$, and ending by 1 .

In order to prove that the family $p(B)$ is free on $\mathbf{C}$, we will use similar arguments as in the case FQSym $/(\mathrm{P}, \mathrm{V}, \mathrm{Dr}, \mathrm{Dd})$.

Proposition 5.3 Let $\sigma$ be in $\Re$. If $\tau$ is in $\Re$, equivalent to $\sigma$ for $(P, V, D r \cup D d)$, and its set of double rises strictly contains the set of double rises of $\sigma$, then $\tau<_{\text {lex }} \sigma$.

\section{Proof:}

Let $\sigma$ and $\tau$ be two permutations satisfying the hypothesis. Let $k$ be the smallest integer such that $k$ is a double rise of $\tau$, and a double descent of $\sigma$ (since the inclusion of double rises set is strict, such a $k$ exists). Since $\sigma$ and $\tau$ are in $\mathfrak{R}$, we have: $\sigma_{(k-1)}=\tau_{(k-1)}=u \infty v$ where $u$ is a word without $\infty$. So, if we apply the $k$-th step of the construction of Françon-Viennot, we have: $\sigma_{(k)}=u \infty k v$, and $\tau_{(k)}=u k \infty v$. Since the first $\infty$ in $\sigma_{(k)}$ will be replaced by a letter greater than $k$, we have $\tau<_{\text {lex }} \sigma$.

Let us denote by $c_{\sigma}^{\tau}$ the number $\left|\left\{s \geq_{P} \sigma \mid s \sim \tau\right\}\right|$. From Proposition 5.3, we have that if $\sigma$ and $\tau$ are in $\mathfrak{D}$, and $\sigma>_{\text {lex }} \tau$, then $c_{\sigma}^{\tau}$ is equal to zero. The $c_{\sigma}^{\sigma}$ are strictly positive, so $\mathbf{C}$ is a free algebra.

\subsection{The quotients of FQSym by $(P \cup D r, V \cup D d)$ or $(P \cup D d, V \cup D r)$}

Thanks to the involutions, these two quotient sets are isomorphic. Note that the equivalence relation ( $\mathrm{P}$ $\cup$ Dr, $\mathrm{V} \cup \mathrm{Dd}$ ) amounts to having the same descent bottoms set, so, hence up to a simple involution, having the same Genocchi set which is the set (see [5]). Thus FQSym $/(\mathrm{P} \cup \mathrm{Dd}, \mathrm{V} \cup \mathrm{Dr}$ ) is isomorphic to NCSF, and so is free. 


\section{References}

[1] G. Duchamp, F. Hivert, and J.-Y. Thibon. Noncommutative Symmetric Functions VI: Free QuasiSymmetric Functions and Related Algebras. International Journal of Algebra and Computation, 12(5):671-717, 2002.

[2] P. Flajolet. Combinatorial aspects of continued fractions. Discrete Mathematics, 32(2):125-161, 1980.

[3] J. Françon and G. Viennot. Permutations selon leurs pics, creux, doubles montées et double descentes, nombres d'Euler et nombres de Genocchi. Discrete Mathematics, 28(1):21 - 35, 1979.

[4] I. Gelfand, D. Krob, A. Lascoux, B. Leclerc, V.S. Retakh, and J.-Y. Thibon. Noncommutative Symmetric Functions. Advances in Mathematics , 112:218-348, 1995.

[5] F. Hivert, J.-C. Novelli, L. Tevlin, and J.-Y. Thibon. Permutation statistics related to a class of noncommutative symmetric functions and generalizations of the Genocchi numbers. Selecta Mathematica, 15:105-119, 2009.

[6] F. Hivert, J.-C. Novelli, and J.-Y. Thibon. The algebra of binary search trees. Theor. Comput. Sci., 339(1):129-165, 2005.

[7] D. Krob, B. Leclerc, and J.-Y. Thibon. Noncommutative Symmetric Functions II: Transformations of alphabets. International Journal of Algebra and Computation, 7(02):181-264, 1997.

[8] D. Krob and J.-Y. Thibon. Noncommutative Symmetric Functions IV: Quantum Linear Groups and Hecke Algebras at q = 0. Journal of Algebraic Combinatorics, 6:339-376, 1997.

[9] A. Lascoux, B. Leclerc, J.-Y. Thibon, et al. The plactic monoid. Algebraic Combinatoric on Words, 2002.

[10] J.-L. Loday and M.-O. Ronco. Hopf Algebra of the Planar Binary Trees. Advances in Mathematics, 139(2):293 - 309, 1998.

[11] C. Malvenuto and C. Reutenauer. Duality between quasi-symmetric functions and the Solomon descent algebra. J. alg., 177:967-982, 1995.

[12] S. Poirier and C. Reutenauer. Algèbres de Hopf de tableaux. Ann. Sci. Math. Québec, 19:79-90, 1995.

[13] N. J. A. Sloane. The On-Line Encyclopedia of Integer Sequences. http://www.research. att.com/ njas/sequences/. 